\title{
THE PECULIAR SOLAR COMPOSITION AND ITS POSSIBLE RELATION TO PLANET FORMATION
}

\author{
J. Meléndez ${ }^{1,5}$, M. AsPlund ${ }^{2}$, B. Gustafsson ${ }^{3}$, and D. Yong ${ }^{4}$ \\ ${ }^{1}$ Centro de Astrofísica da Universidade do Porto, Rua das Estrelas, 4150-762 Porto, Portugal; jorge@astro.up.pt \\ 2 Max-Planck-Institut für Astrophysik, Karl-Schwarzschild-Str. 1, Postfach 1317, D-85741 Garching, Germany \\ ${ }^{3}$ Institutionen för fysik och astronomi, Uppsala universitet, Box 515, SE-75120 Uppsala, Sweden \\ ${ }^{4}$ Research School of Astronomy \& Astrophysics, Australian National University, Mount Stromlo Observatory, Cotter Road, Weston Creek, ACT 2611, Australia \\ Received 2009 August 1; accepted 2009 September 11; published 2009 September 25
}

\begin{abstract}
We have conducted a differential elemental abundance analysis of unprecedented accuracy $(\sim 0.01$ dex $)$ of the Sun relative to 11 solar twins from the Hipparcos catalog and 10 solar analogs from planet searches. We find that the Sun shows a characteristic signature with a $\approx 20 \%$ depletion of refractory elements relative to the volatile elements in comparison with the solar twins. The abundance differences correlate strongly with the condensation temperatures of the elements. This peculiarity also holds in comparisons with solar analogs known to have close-in giant planets while the majority of solar analogs found not to have such giant planets in radial velocity monitoring show the solar abundance pattern. We discuss various explanations for this peculiarity, including the possibility that the differences in abundance patterns are related to the formation of planetary systems like our own, in particular to the existence of terrestrial planets.
\end{abstract}

Key words: planetary systems - solar system: formation - stars: abundances - Sun: abundances

\section{INTRODUCTION}

Studies of extra-solar planetary systems and the possibility of life in the universe depend fundamentally on whether the Sun and its planets are typical (Gustafsson 2008; Beer et al. 2004; Robles et al. 2008; Seager 2003). Previous chemical composition studies have concluded that the Sun is a typical star (Gustafsson 1998, 2008; Robles et al. 2008). Besides an increased likelihood for stars with higher overall metallicity of hosting giant planets, no definitive chemical differences have been established between stars with and without known planets (Gonzales 1997; Ecuvillon et al. 2006; Udry \& Santos 2007). Previous studies on chemical abundance anomalies in the Sun as compared with solar-like stars have been inconclusive due to the relatively large ( $\gtrsim 0.05 \mathrm{dex})$ remaining systematic errors (Gustafsson 2008; Robles et al. 2008; Reddy et al. 2003). In such studies, the selection of stars to which the Sun is compared is crucial. Different categories of solar-like stars can be identified. "Solar-type stars" range from late F to early K, "solar analogs" include only G0-G5 dwarfs, and stars almost identical to the Sun are called "solar twins" (Cayrel de Strobel 1996). In modern stellar chemical analyses, the error budget is dominated by systematic errors in the model atmospheres and modeling of stellar spectra (Asplund 2005). The highest possible accuracy in the analysis of the Sun relative to stars can be achieved in comparisons relative to solar twins, since the model errors will cancel to a large extent and effects of possible systematic errors in temperature scale and absolute abundances are minimized.

\section{OBSERVATIONS AND ANALYSIS}

Our sample comprises 11 solar twins and 10 solar analogs. The solar twins were selected from more than 100,000 stars in the Hipparcos catalog by employing precise colortemperature relations, trigonometric parallaxes, and age indicators (Meléndez et al. 2006; Meléndez \& Ramírez 2007). They lie within a distance of $75 \mathrm{pc}$ from the Sun and belong to the

\footnotetext{
5 Visiting Astronomer, Magellan Telescopes, Las Campanas Observatory, Chile, and W.M. Keck Observatory, Manua Kea, Hawaii.
}

Galactic thin disk population. No giant planets are known around our solar twins because most of them have not been searched for planets. The solar analogs were selected from radial velocity planet surveys (Udry \& Santos 2007). The Sun (reflected light from the asteroid Vesta), solar analogs, and solar twins were observed with the MIKE spectrometer at the Clay $6.5 \mathrm{~m}$ Magellan telescope at Las Campanas Observatory in Chile, at high spectral resolution $(R=\Delta \lambda / \lambda=65,000)$ and very high signal-to-noise ratio $(\mathrm{S} / \mathrm{N} \sim 450$ per pixel at $600 \mathrm{~nm})$ through the wavelength range 340-1000 nm. Example Magellan spectra of solar twins are presented in Figure 1. Finally, one of our twins (HIP $79672=18$ Sco) was also observed with the HIRES spectrograph on the Keck I telescope to yield an even higher quality spectrum with $\mathrm{S} / \mathrm{N} \approx 450$ and $R=100,000$; a solar spectrum (asteroid Ceres) was also obtained with identical setup.

A model independent analysis (Meléndez et al. 2006; Meléndez \& Ramírez 2007) indicates that the twins have effective temperatures within $75 \mathrm{~K}$ of the Sun, logarithmic surface gravities within $0.10 \mathrm{dex}$, and logarithmic iron abundances $[\mathrm{Fe} / \mathrm{H}]$ within $0.07 \mathrm{dex}\left(\right.$ where $[\mathrm{A} / \mathrm{B}]=\log _{10}\left(N_{A} / N_{B}\right)_{\text {star }}-$ $\left.\log _{10}\left(N_{A} / N_{B}\right)_{\odot}\right)$. These indications were confirmed by a detailed model-atmosphere analysis. The final adopted effective temperatures were obtained from the excitation equilibrium of $\mathrm{Fe}$ I, while the surface gravities were determined from the ionization equilibrium of $\mathrm{Fe}$ I/Fe II. We have achieved an accuracy in $[\mathrm{X} / \mathrm{Fe}]$ at the level of $0.01 \mathrm{dex}$ for the solar twins (e.g., the star-to-star scatter in $[\mathrm{Cr} / \mathrm{Fe}]$ is only $\sigma=0.009 \mathrm{dex}$ ). For the solar analogs, high precision abundances are also obtained due to their similarity to the Sun, although not as high as for the solar twins. The observed scatter in relative abundances is fully consistent with the predicted error from the quality of the spectra and the remaining uncertainties in the stellar parameters.

\section{RESULTS}

Our results reveal that the solar chemical abundances relative to iron depart from the mean abundance ratios in the solar twins (Figure 2). The Sun is enriched by $\sim 0.05$ dex in most of the lighter elements, while other elements like aluminum are 


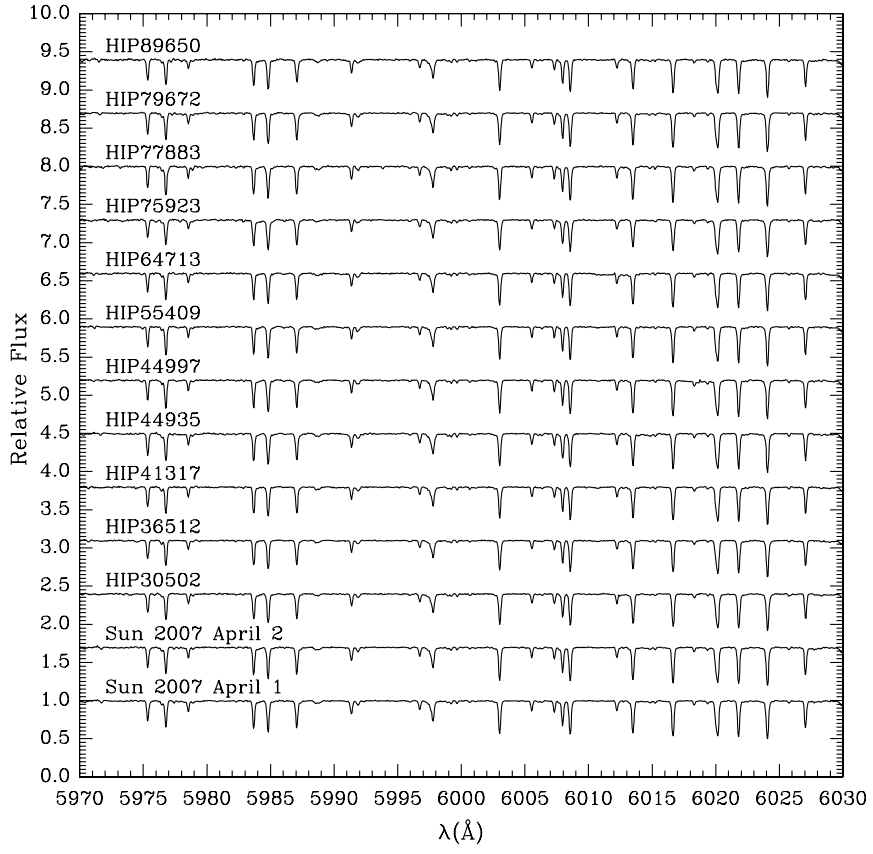

Figure 1. High-quality $(\mathrm{S} / \mathrm{N} \sim 450)$ MIKE spectra of the Sun and solar twins in the region 5970-6030 A.

deficient by $\sim 0.03$ dex, thus the offset between the elements that are depleted and enhanced is $\sim 0.08$ dex $(20 \%)$. In Figure 3, the abundance differences are shown versus the $50 \%$ condensation temperature $T_{\text {cond }}$ (Lodders 2003) for a solar-system composition gas. Volatile elements (low $T_{\text {cond }}$ ) are more abundant in the Sun relative to the twins while elements that easily form dust (elements with high $T_{\text {cond }}$, i.e., refractories) are underabundant. A very similar pattern, albeit of much greater amplitude, is seen in the interstellar medium (ISM) due to dust condensation (Savage $\&$ Sembach 1996), as well as in $\lambda$ Bootis stars and some highly evolved stars through the accretion of dust-cleansed gas (Venn \& Lambert 1990). The correlation with $T_{\text {cond }}$ shown in Figure 3 is highly significant: the Spearman correlation coefficient is $r_{\mathrm{S}}=-0.91$ and an arrangement of this type has a probability of $\sim 10^{-9}$ to happen by chance. The scatter around the mean trend in Figure 3 is only 0.01 dex. On a star-by-star basis, only 1-3 solar twins resemble the Sun chemically (Figure 4). In fact, the probability of obtaining the same order between abundances differences for the four degrees of volatility for 9 (and very nearly 10) out of 11 stars as a result of random errors is only $2 \times 10^{-11}$. Previous attempts to trace $T_{\text {cond }}$-dependent abundance differences for stars known to host giant planets have not convincingly demonstrated significant differences (Udry \& Santos 2007), probably due to the high accuracy needed.

The results for the solar analogs are shown in Figure 5. The solar analogs with detected giant planets have an abundance pattern similar to the solar twins, while the solar analogs without detected giant planets are more similar to the Sun.

In order to confirm that our results are robust, we have checked our abundance scale using spectra obtained with another telescope and spectrograph. We repeated our highly differential abundance analysis for the Sun and the solar twin HIP 79672, using a HIRES/Keck spectrum. Our Keck-based results confirm the separation between volatile and refractory elements found with the MIKE data. The $[\mathrm{X} / \mathrm{Fe}]$ abundance ratios of the HIRES analysis are in excellent agreement with our MIKE results. In particular, the relative abundances of

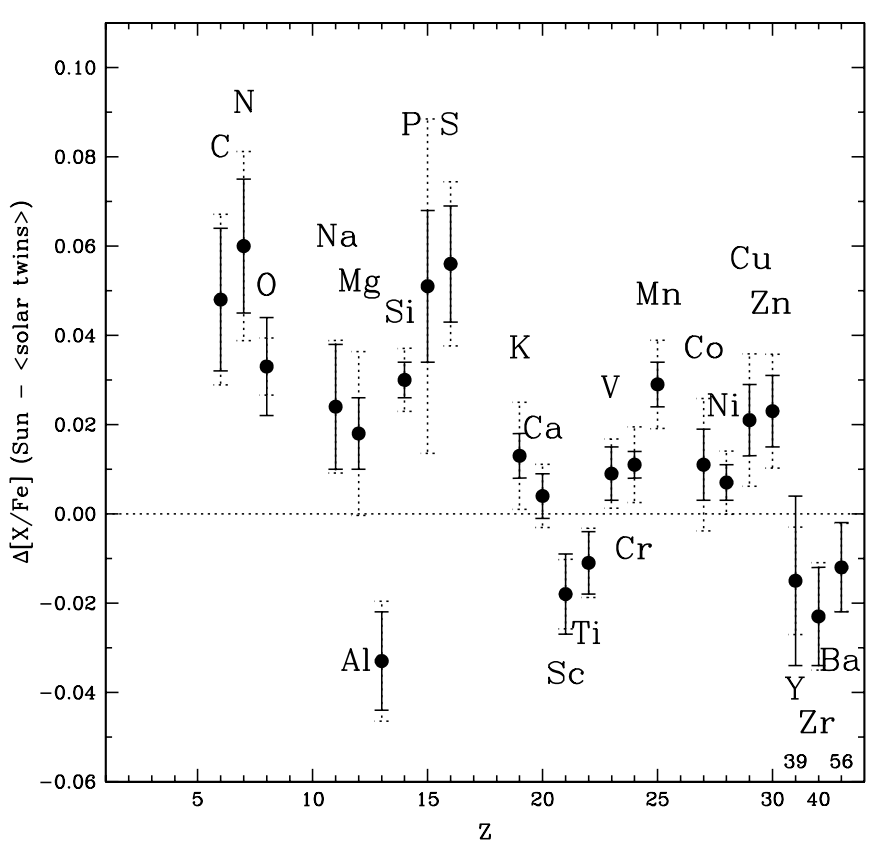

Figure 2. Differences between $[X / F e]$ of the Sun and the mean values in the solar twins as a function of atomic number $Z$. For clarity, the elements $\mathrm{Y}(Z=$ $39), \mathrm{Zr}(Z=40)$, and $\mathrm{Ba}(Z=56)$ have been included after $\mathrm{Zn}$. Observational $1 \sigma$ errors in the relative abundances (including observational errors in both the Sun and solar twins) are shown with dotted error bars, while the $1 \sigma$ errors in the mean abundance of the solar twins are shown with solid error bars.

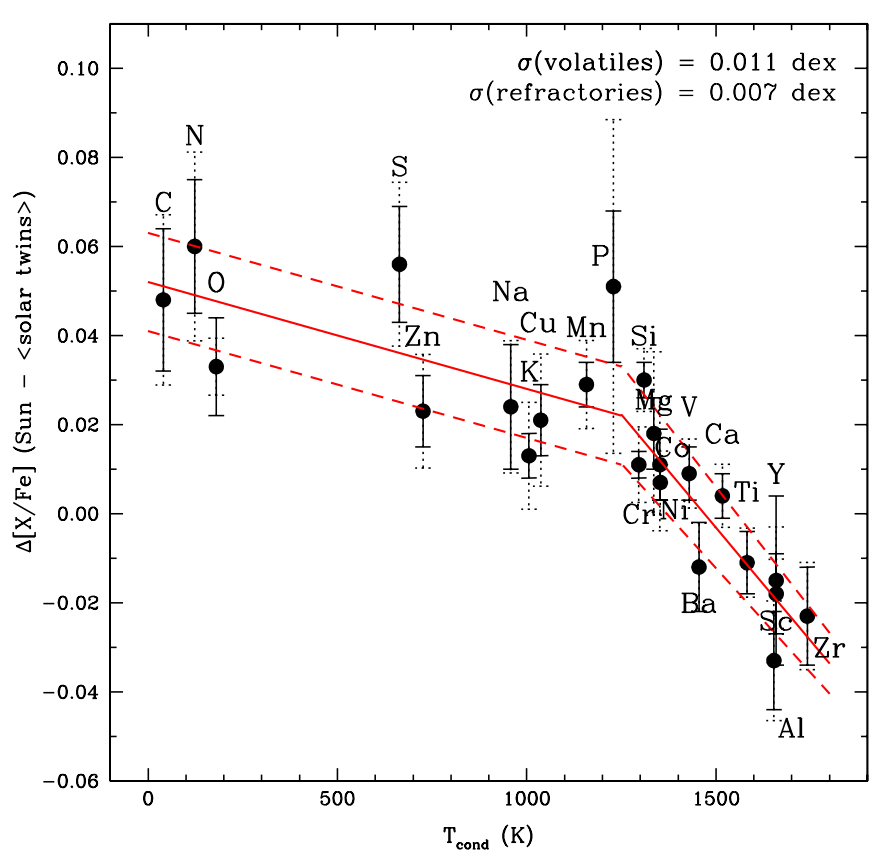

Figure 3. Differences between $[\mathrm{X} / \mathrm{Fe}]$ of the Sun and the mean values in the solar twins as a function of $T_{\text {cond }}$. The abundance pattern shows a break at $T_{\text {cond }} \sim 1200 \mathrm{~K}$. The solid lines are fits to the abundance pattern, while the dashed lines represent the standard deviation from the fits. The low elementto-element scatter from the fits for the refractory $(\sigma=0.007 \mathrm{dex})$ and volatile $(\sigma=0.011 \mathrm{dex})$ elements confirms the high precision of our work. The zero point for the differences in relative chemical abundances depends on the adopted reference element, which here is Fe; the volatiles would appear normal while the refractories more depleted had we instead selected to use C. Error bars as in Figure 2.

volatiles like carbon, oxygen, and zinc, agree to within 0.003 dex between the HIRES and MIKE data. This demonstrates that our abundance scale is robust, and that the quality of the 


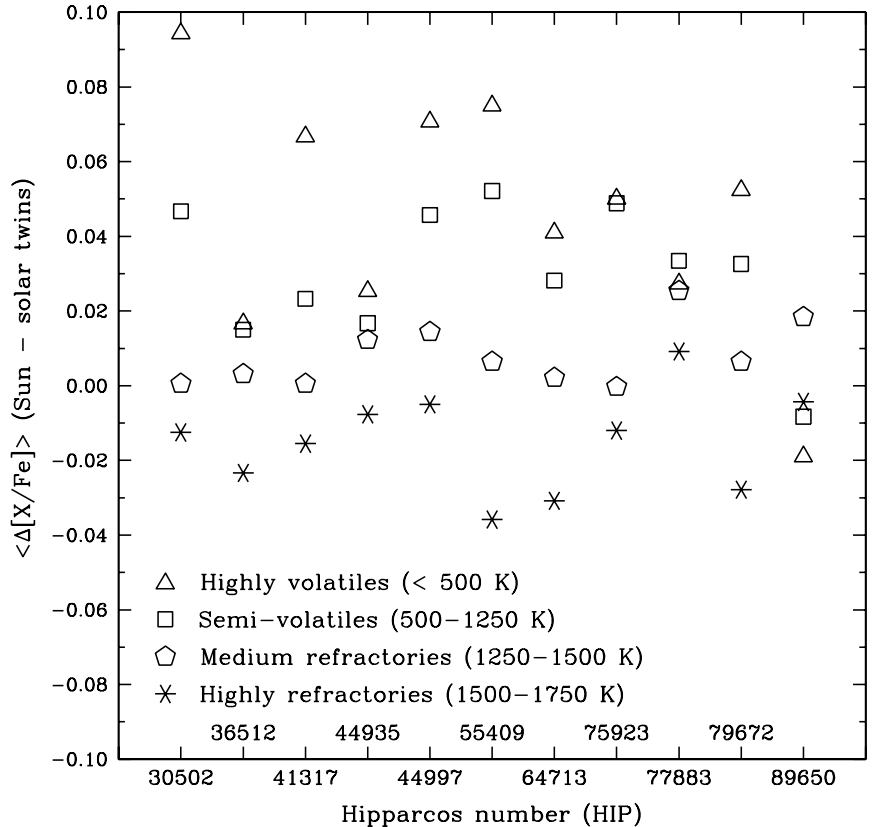

Figure 4. Mean $[\mathrm{X} / \mathrm{Fe}]$ ratios observed in the solar twins for highly volatile (triangles), semi-volatile (squares), medium refractory (pentagons), and highly refractory (stars) elements, for the solar twins. Most twins show similar trends with $T_{\text {cond }}$ with the highly refractory elements $\left(1500<T_{\text {cond }}<1750 \mathrm{~K}\right)$ well separated from the highly volatile elements $\left(T_{\text {cond }}<500 \mathrm{~K}\right)$ and in between the semi-volatile $\left(500<T_{\text {cond }}<1250 \mathrm{~K}\right)$ and medium refractory $\left(1250<T_{\text {cond }}<1500 \mathrm{~K}\right)$ elements. This separation between refractories and volatiles on a star-by-star basis further emphasizes that the abundance differences are indeed real.

MIKE spectra is high enough to allow our conclusion that the refractories in the Sun are depleted with respect to the volatiles.

We are not aware of any systematic errors that would, if corrected for, significantly reduce these differences between the Sun and the twins. An error in the temperature scale would lead to even larger departures in $[\mathrm{X} / \mathrm{Fe}]$ but not generate the consistent pattern found here.

\section{THE ORIGIN OF THE SOLAR DEPARTURES}

In spite of the suggestions above that the solar characteristics could be related to dust condensation, we shall here first discuss other possible explanations.

1. Galactic-evolution effects. Perhaps the solar abundance pattern reflects the Galactic chemical evolution and the varying composition in the Milky Way ISM when the Sun was formed. The $[\alpha / \mathrm{Fe}]$ ratio ( $\alpha$ representing $\mathrm{O}, \mathrm{Ne}, \mathrm{Mg}$, $\mathrm{Si}, \mathrm{Ca}, \mathrm{S}$, and $\mathrm{Ti}$ ) was higher earlier in the history of the Galactic disk, due to the increasing role of SN Ia relative to that of SN II in enriching the ISM. The solar twins have iron abundances similar to solar. If the metallicity of the Sun is unusually high for its age, most twins could be younger and have lower $[\alpha / \mathrm{Fe}]$. The Sun is, however, not so metal rich for its age (Fuhrmann 2004; Holmberg et al. 2009) and it is unlikely that the variation of $[\alpha / \mathrm{Fe}]$ with metallicity is large enough for this hypothesis to work (Bensby \& Feltzing 2006; Chen et al. 2002; Nissen et al. 2004). Besides, the abundances relative to $\mathrm{Fe}$ for the volatile elements $\mathrm{C}, \mathrm{S}$, and $\mathrm{Zn}$ as well as the refractory element $\mathrm{Si}$ all show no trend with metal content around solar metallicity. One direct way of testing this hypothesis would be to compare the ages of the twins to the Sun. Available methods for estimating

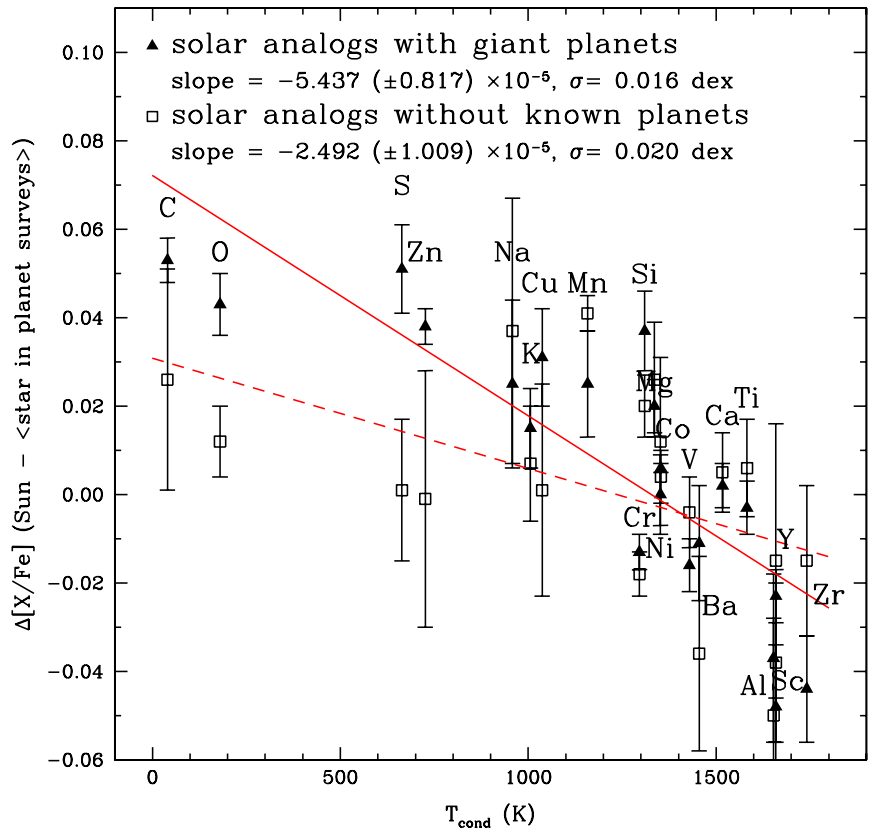

Figure 5. Differences between $[\mathrm{X} / \mathrm{Fe}]$ of the Sun and the mean values in the solar analogs with (filled triangles) and without (open squares) detected planets, as a function of their $T_{\text {cond }}$. The planet hosting stars show a strong trend with $T_{\text {cond }}$ almost identical to that seen in the solar twins (Figure 3). The correlation is highly statistically significant $\left(r_{\mathrm{S}}=-0.91\right)$ with a probability of having this correlation by pure chance of $\sim 10^{-8}$. Solar analogs without planets show a much shallower slope and a much weaker correlation. Linear fits to both samples are shown. The slope of the stars with planets is significant at the $7 \sigma$ level, while the slope of the stars without planets is lower and significant only at the $2 \sigma$ level.

stellar ages (Meléndez et al. 2006; Meléndez \& Ramírez 2007) applied to the twins reveals that their median age is 4.1 Gyr. Thus, the peculiar abundance pattern of the Sun is not the result of large age differences between the Sun and the twins.

Wielen et al. (1996) suggested that the Sun may have migrated from an inner Galactic orbit. If there were radial abundance gradients, systematic differences between the Sun and the twins may arise. However, the observed gradients in $[\mathrm{O} / \mathrm{Fe}]$ for young thin disk objects are not steep enough (Esteban et al. 2005; Przybilla et al. 2008), and Galactic chemical evolution models (Chiappini et al. 2001) do not suggest any substantially steeper gradient 5 Gyr ago.

2. Supernova pollution. Another possibility is that the protosolar cloud happened to be more polluted by a local supernova than other star formation regions in the Galaxy, although this hypothesis does not explain why the Sun, and not the twins, happens to be affected. However, the peculiar solar abundance pattern cannot be explained by an offset of SN II relative to SN Ia yields (Woosley \& Weaver 1995; Thielemann et al. 2002). This argument also negates the solar migration scenario. Finally, a suggestion that the presence of ${ }^{60} \mathrm{Fe}$ in meteorites proves that the proto-solar nebula was affected by a nearby supernova explosion was recently found less likely (Williams 2009).

3. Early dust separation. One possible explanation is that the Sun was formed in an interstellar cloud where dust had been blown away beforehand, e.g., by luminous massive stars. Another possibility could be that the late formation process of the Sun included accretion of gas that was metal poor due to dust separation by radiation pressure, although it is questionable whether the accreting proto-Sun was 
efficient enough in this respect. Neither of these scenarios can explain why this happened to the Sun but not the twins nor can it explain the correlation with the presence of closein giant planets (explanation (5) below).

4. Dust separation during the formation of terrestrial planets. A fascinating possibility is that the composition departures of the Sun are related to its properties as a planet host. A particularly striking circumstance is that the inner solarsystem planets and meteorites are enriched in refractories compared to volatiles (Palme 2000). The abundance pattern of these bodies is almost a mirror image of the solar pattern relative to the solar twins (Ciesla 2008; Alexander et al. 2001). Also, a radial gradient exists, with greater enhancement of refractories at smaller heliocentric distances (Palme 2000; Bottke et al. 2006). The break in the chemical abundance pattern at $T_{\text {cond }} \approx 1200 \mathrm{~K}$ (Figure 3 ), suggests that the volatiles retained their original abundances both in the Sun and the solar twins. Such temperatures are only encountered in the inner parts $(<3 \mathrm{AU})$ of protoplanetary disks (Ciesla 2008), which also suggests that the abundance pattern is related to the presence of terrestrial planets.

The amount of dust-depleted gas required to explain the solar abundances depends sensitively on the timing of the accretion. The mass fraction of the solar convection zone dropped from $100 \%$ to $38 \%$ in $10 \mathrm{Myr}$, and to $2 \%$ after another $20 \mathrm{Myr}$, thereafter remaining largely constant (D'Antona \& Mazzitelli 1994). Assuming that the depletion signature was imprinted once the convection zone reached its present size, the removal of $\sim 2 \times 10^{28} \mathrm{~g}$ of refractories from the accreting gas would be required in order to accomplish the observed $\sim 10 \%$ reduction of refractory elements. This value is similar to the combined mass of refractories locked up in Mercury, Venus, Earth, and Mars, which is $\sim 8 \times 10^{27} \mathrm{~g}\left(1.3 M_{\oplus}\right)$. The formation of large $(\sim 10 \mathrm{~km})$ bodies and embryos of terrestrial planets in the proto-solar disk is estimated to have an efficiency of 30\%-50\% (Alexander et al. 2001). Thus, it is tempting to speculate that the formation of the terrestrial planets might have given the Sun its special surface composition. The disk masses during the T Tauri phase are $\sim 0.02 M_{\odot}$ but values up to $1 M_{\odot}$ are possible (Beckwith et al. 1990; Calvet et al. 2000), thus enough material would be available to change the solar photospheric composition. However, there is a problem with timescales since proto-planetary disks are observed to have typical lifetimes $<10 \mathrm{Myr}$ (Calvet et al. 2000; Sicilia-Aguilar et al. 2006). Yet, gas accretion onto 10-25 Myr old stars has been detected but is rare: only $1 \%$ of stars at an age of 13 Myr show signs of accretion (Currie et al. 2007; White \& Hillebrand 2005). One possibility to explain the chemical differences with the solar twins is that they did not form terrestrial planets or, if they did, the subsequent gas accretion occurred when the stellar convection zone was still deep and the planetary signatures thus washed out, while the Sun retained its planetary disk longer. A less likely explanation is that all solar twins formed terrestrial planets and like the Sun lost their gas disks when their convection zones were deep, but accreted their terrestrial planets later (after $30 \mathrm{Myr}$ ) when the convection zones were thin, thus enriching the solar twins in refractory elements. There are reasons to believe that thermohaline convection, generated by the inverse $\mu$-gradient, would then have quickly erased the higher metallicity of the stellar convection zone (Vauclair 2004).

5. The role of giant planets. The abundance pattern could also be the result of the formation of giant planets. The cores of Jupiter, Saturn, Uranus, and Neptune containing heavy metals in the form of rocks and ices are estimated to have a combined mass of $\sim 30 M_{\oplus}$ (Guillot 2005). Thus, the accretion could have occurred earlier, which would alleviate the problem with disk lifetimes. If so, stars with giant planets would be expected to show a chemical abundance pattern more similar to solar. Our sample of 10 solar analogs from planet surveys includes four solar analogs with giant planets, while for the other six no planets have been detected yet. As shown in Figure 5, all solar analogs with giant planets differ from the Sun but closely resemble most solar twins. Thus, the odd solar composition is not due to giant planets as such. On the other hand, only two of the six solar analogs without close-in giant planets have abundances that differ significantly from the solar pattern. The fraction of stars with the solar pattern seems thus tentatively related to the presence of giant planets on close orbits: $\approx 0 \%$ when having such planets, $\approx 20 \%$ for solar-type stars in general, and $\approx 50 \%-70 \%$ without close-in giant planets. No doubt, the statistics have to be improved considerably, but the numbers are clearly tantalizing.

\section{CONCLUSIONS}

We conclude that the Sun is unusual albeit not unique in its detailed elemental abundance pattern. In particular, it has a chemical composition that is more affected by dust condensation than most other similar stars, such as most solar twins and all solar analogs with discovered giant planets. This may be purely circumstantial. For instance, the cloud in which the Sun was formed may first have been cleansed from dust by radiation from hot luminous stars to a higher degree than happened where most other solar-type stars were formed. However, if an explanation of less ad hoc character is searched for, it is natural to explore whether the solar composition could be related to the way the formation of the planets in the solar system occurred. A reasonable hypothesis is then that the accretion onto the Sun of the proto-planetary solar nebula, chemically affected by dust condensation and planet formation, was delayed long enough for the cleansed gas to significantly affect the composition of the solar convection zone. Another possibility is that the early Sun was never fully convective, which would make it easier to imprint a dust-cleansed abundance signature (Nordlund 2009). This scenario is supported by the dynamical star formation calculations with a time-dependent convection treatment of Wuchterl \& Tscharnuter (2003) and Wuchterl \& Klessen (2001).

The fact that stars with known close giant planets do not show the solar pattern suggests that their gas disks for some reason were accreted earlier when the stellar convection zones were so deep that the planetary signatures in the infalling gas disks were erased, and/or that the key factor behind the solar pattern is the formation and non-accretion of inner terrestrial planets. This would imply that solar-like stars with planetary systems similar to our own are a relatively rare occurrence. Before firm conclusions can be drawn regarding the effects and frequency of planet formation, accurate analyses of significantly larger samples of solar-like stars with and without known giant planets are important. It is reassuring that an independent analysis of 22 northern solar twins confirm our findings both in terms of the magnitude of the abundance difference between volatiles and 
refractories and the frequency of stars showing the peculiar solar abundance pattern (Ramirez et al. 2009). Our findings provide strong impetus to improve the abundance accuracy of late-type stars to the 0.01 dex level by removing the remaining systematic errors in the modeling of stellar atmospheres and line formation (Asplund 2005; Asplund et al. 2009), which have obscured the planetary signatures in the stellar compositions until now. It is an enthralling prospect to be able to identify stars with planetary systems similar to the solar system by means of their abundance patterns.

Our results are based on observations made using Australian time at the Clay $6.5 \mathrm{~m}$ Magellan telescope at Las Campanas Observatory in Chile. Travel support was provided by the Australian Access to Major Facilities Programme (06/07-O11). We thank A. Alves-Brito for help with the data reduction; J. Johnson for her NH line list; W. Lyra, M. Davies, and T. Henning for discussions on disk lifetimes, planetary connections, and effects of stellar passages; A. Serenelli, C. Straka, S. Vauclair, and A. Weiss for discussions on stellar evolution and diffusion; and Åke Nordlund for discussions on early stellar evolution and planetary formation.

We acknowledge support from the Portuguese FCT (project PTDC/CTE-AST/65971/2006, and Ciencia 2007 program), the Australian Research Council, and the Swedish Research Council.

Facilities: GMT (MIKE), Keck I (HIRES)

\section{REFERENCES}

Alexander, C. M. O’D., Boss, A. P., \& Carlson, R. W. 2001, Science, 293, 64 Asplund, M. 2005, ARA\&A, 43, 481

Asplund, M., Grevesse, N., Sauval, A. J., \& Scott, P. 2009, ARA\&A, 47, 481

Beckwith, S. V. W., Sargent, A. I., Chini, Rolf, S., \& Guesten, R. 1990, AJ, 99 924

Beer, M. E., King, A. R., Livio, M., \& Pringle, J. E. 2004, MNRAS, 354, 763 Bensby, T., \& Feltzing, S. 2006, MNRAS, 367, 1181

Bottke, W. F., Nesvorný, D., Grimm, R. E., Morbidelli, A., \& O’Brien, D. P. 2006, Nature, 439, 821
Calvet, N., Hartmann, L., \& Strom, S. E. 2000, Protostars and Planets, IV, ed. V. Mannings, A. P. Boss, \& S. S. Russell (Tucson, AZ: Univ. Arizona Press), 377

Cayrel de Strobel, G. 1996, A\&AR, 7, 243

Chen, Y. Q., Nissen, P. E., Zhao, G., \& Asplund, M. 2002, A\&A, 390, 225

Chiappini, C., Matteucci, F., \& Romano, D. 2001, ApJ, 554, 1044

Ciesla, F. J. 2008, Meteorit. Planet. Sci., 43, 639

Currie, T., Kenyon, S. J., Balog, Z., Bragg, A., \& Tokarz, S. 2007, ApJ, 669 L33

D’Antona, F., \& Mazzitelli, I. 1994, ApJS, 90, 467

Ecuvillon, A., Israelian, G., Santos, N. C., Mayor, M., \& Gilli, G. 2006, A\&A, 449,809

Esteban, C., et al. 2005, ApJ, 618, L95

Fuhrmann, K. 2004, Astron. Nachr., 325, 3

Gonzalez, G. 1997, MNRAS, 285, 403

Guillot, T. 2005, Annu. Rev. Earth Planet. Sci., 33, 493

Gustafsson, B. 1998, Space Sci. Rev., 85, 419

Gustafsson, B. 2008, Phys. Scr. T, 130, 014036

Holmberg, J., Nordström, B., \& Andersen, J. 2009, A\&A, 501, 941

Lodders, K. 2003, ApJ, 591, 1220

Meléndez, J., Dodds-Eden, K., \& Robles, J. A. 2006, ApJ, 641, L133

Meléndez, J., \& Ramírez, I. 2007, ApJ, 669, L89

Nissen, P. E., Chen, Y. Q., Asplund, M., \& Pettini, M. 2004, A\&A, 415, 993

Nordlund, A. 2009, ApJ, submitted (arXiv:0908.3479)

Palme, H. 2000, Space Sci. Rev., 92, 237

Przybilla, N., Nieva, M.-F., \& Butler, K. A 2008, ApJ, 688, L103

Ramírez, I., Meléndez, J., \& Asplund, M. 2009, A\&A, submitted

Reddy, B. E., Tomkin, J., Lambert, D. L., \& Allende Prieto, C. 2003, MNRAS, 340, 304

Robles, J. A., et al. 2008, ApJ, 684, 691

Savage, B. D., \& Sembach, K. R. 1996, ARA\&A, 34, 279

Seager, S. 2003, Earth Planet. Sci. Lett., 208, 113

Sicilia-Aguilar, A., et al. 2006, AJ, 132, 2135

Thielemann, F.-K., et al. 2002, Ap\&SS, 281, 25

Udry, S., \& Santos, N. C. 2007, ARA\&A, 45, 397

Vauclair, S. 2004, ApJ, 605, 874

Venn, K. A., \& Lambert, D. L. 1990, ApJ, 363, 234

White, R. J., \& Hillenbrand, L. A. 2005, ApJ, 621, L65

Wielen, R., Fuchs, B., \& Dettbarn, C. 1996, A\&A, 314, 438

Williams, J. P. 2009, in Les Houches Winter School Physics and Astrophysics of Planetary Systems, EDP Sciences, (EAS Publication Series), in press (arXiv:0808.2506)

Woosley, S. E., \& Weaver, T. A. 1995, ApJS, 101, 181

Wuchterl, G., \& Klessen, R. S. 2001, ApJ, 560, L185

Wuchterl, G., \& Tscharnuter, W. M. 2003, A\&A, 398, 1081 